\title{
Prevalence and Predicting Factors for Anxiety in Thai Women with Abnormal Cervical Cytology Undergoing Colposcopy
}

\author{
Tanut Jerachotechueantaveechai ${ }^{1}$, Kittipat Charoenkwan ${ }^{1 *}$, Nahathai \\ Wongpakaran ${ }^{2}$
}

\begin{abstract}
Aim: To compare prevalence of anxiety in women with abnormal cervical cytology (Pap) undergoing colposcopy to that of women attending the outpatient clinic for check-up and to examine predicting factors. Materials and Methods: In this cross-sectional analytical study, 100 women with abnormal cervical cytology (abnormal Pap group) and 100 women who attended our outpatient clinic for check-up (control group) were recruited from June 2013 to January 2014. The Hospital Anxiety and Depression Scale (HADS) was employed to determine anxiety in the participants with the score of $\geq 11$ suggestive of clinically significant anxiety. The prevalence of anxiety and the mean HADS scores for anxiety were compared between the groups. For those with abnormal Pap, association between clinical factors and anxiety was assessed. A p-value of $<0.05$ was considered significant. Results: Median age was different between the groups, 44.0 years in the abnormal Pap group and 50.0 years in the control group ( $p=0.01)$. The proportion of participants who had more than one sexual partner was higher in the abnormal Pap group, 39.2\% vs. $24.7 \%(\mathrm{p}=\mathbf{0 . 0 3})$ and the prevalence of anxiety was significantly higher $14 / 100(14.0 \%)$ vs. $3 / 100(3.0 \%)(p<0.01)$. The prevalence of depression was comparable between the groups. The mean HADS scores for anxiety and depression subscales were significantly higher in the abnormal Pap group, 6.6 vs. $4.8(\mathrm{P}<0.01)$ and 3.9 vs. $3.1(\mathrm{p}=0.05)$, respectively. For the abnormal Pap group, no definite association between clinical factors and anxiety was demonstrated. Conclusions: The prevalence of anxiety in women with abnormal Pap awaiting colposcopy was significantly higher than that of normal controls. Special attention including thorough counselling, with use of information leaflets and psychological support, should be directed to these women.
\end{abstract}

Keywords: Abnormal cervical cytology - anxiety - hospital anxiety - depression scale - Pap smear - prevalence

Asian Pac J Cancer Prev, 16 (4), 1427-1430

\section{Introduction}

Over the past 50 years, the incidence and mortality of cervical cancer has decreased by 75 percent in developed countries because of the availability of screening program for cervical precancer and human papilloma virus (HPV) vaccination. In developing countries, however, cervical cancer remains the second most common type of cancer and cause of cancer death in women. This is most likely due to the lack of effective screening program for precancer (Quinn et al., 1999; Willoughby et al., 2006). Cervical cytology through the use of Papanicolaou (Pap) smear has been the mainstay for cervical cancer screening. With this technique, abnormal dysplatic cells can be detected early and proper management can be made before the dysplasia developing into cancer. Approximately 6-7\% of all Pap smear are reported as abnormal. As a result, $4-5 \%$ of women who have Pap smear need further diagnostic evaluation. Colposcopy (visualization of the cervix through magnifying binocular) with directed cervical biopsy is the most frequently performed procedures in this situation. Significant level of anxiety and stress can be expected among these women due to uncertainty in status of cancer diagnosis and also in the process of colposcopy (Marteau et al., 1990; Kavanagh and Broom, 1997). This could result in adverse consequences that include failure to comply with colposcopic appointment and increased pain and discomfort during colposcopic procedure.

The objectives of this study were to compare prevalence of anxiety symptoms in women with abnormal cervical cytology undergoing colposcopy to that of women attending the outpatient clinic for check-up and to examine predicting factors for anxiety in the women with abnormal cervical cytology.

\section{Materials and Methods}

From June 2013 to January 2014, 100 consecutive women, aged 18-65 years old, with abnormal Pap test results, who came to our clinic for colposcopic 


\section{Tanut Jerachotechueantaveechai et al}

examination (abnormal Pap group) and 100 women who attended our outpatient clinic during the same period for check-up (control group) were recruited for this crosssectional analytical study. Exclusion criteria included not being able to communicate in Thai. Institutional Review Board approved this study. Verbal and written informed consents were obtained from all participants prior to participation. Demographic and clinical data were collected. The self-administered questionnaire, the Thai version of Hospital Anxiety and Depression Scale (HADS) (Zigmond and Snaith, 1983; Nilchaikovit et al., 1996), was used to determine anxiety and depression symptoms of the participants. The questionnaires comprised 14 items, seven for anxiety and seven for depression. Each item was constructed as a four-point Likert scale ranging from 0 (the least anxious/depressed) to 3 (the most anxious/ depressed). At the end of the assessment, the total scores for anxiety subscale (HADS-A) and depression subscale (HADS-D) were considered separately. For each subscale, the score of 8-10 was suggestive of borderline anxiety or depressive symptoms while the score of $>11$ indicated clinical anxiety or depression (or significant anxiety or depression symptoms) (Nilchaikovit et al., 1996). Internal consistency of the subscales was examined by using the Cronbach's alpha statistics. Each patient completed the questionnaires independently by herself while waiting for the colposcopic procedure (abnormal Pap group) or waiting to be seen by the physicians (control group). The primary outcome of this study was anxiety symptoms indicated by the HADS-A score of $>11$. The secondary outcome was depression according to the HADS-D score of $>11$. The prevalence of anxiety and depression and mean HADS scores for anxiety and depression were compared between the study groups by the use of chi-squared test or Fisher's exact test and student's t-test, respectively. Multivariable analysis using logistic regression was performed to examine the association between demographic/clinical factors including study group (abnormal Pap vs control), age, marital status, religion, alcohol consumption, co-morbidity, salary, and education and anxiety. For those with abnormal Pap smear, association of clinical and pathological factors with anxiety was examined. Statistical analysis was performed by using Stata ${ }^{\circledR}$ program version 12 (StataCorp LP, College Station, Texas, USA). The p-value of $<0.05$ was considered significant.

\section{Results}

Two hundred women, 100 in the study group and 100 in the control group, participated in this study. Median age was 44 years (range 16-66 years) in the abnormal Pap group and 50 years (range 17-78 years) in the control group $(\mathrm{p}=0.01)$. Median number of sexual partner was 1 in both groups (range 1-5 in abnormal Pap group and 1-3 in control group). Demographic data for both groups are presented in Table 1. The two study groups were similar with respect to parity, marital status, smoking, alcohol consumption, co-morbidity, history of cancer, family history of cancer, availability of caregiver, religion, education, and salary. However, participants in the control group were significantly older and higher proportion of participants in the abnormal Pap group had more than one sexual partner. For participants in the abnormal Pap group, waiting duration from receiving Pap result and colposcopy was $1-2$ month in $47.4 \%$, less than 1 month in $33.3 \%$, and more than 2 months in $19.2 \%$.

The Cronbach's alpha coefficients of internal consistency of the HADS questionnaires were 0.82 for the anxiety subscale ( 0.82 for the abnormal Pap group and 0.80 for the control groups) and 0.71 for the depression subscale ( 0.72 for the abnormal Pap group and 0.70 for the control group). Table 2 compares rate of anxiety and depression and HADS scores for anxiety and depression between the two study groups. The prevalence of anxiety was significantly higher in the abnormal Pap

\section{Table 1. Demographic Data}

\begin{tabular}{|c|c|c|c|c|}
\hline Characteristics & $\mathrm{N}(\%)$ & $\begin{array}{l}\text { Normal } \\
\text { control } \\
\mathrm{N}(\%)\end{array}$ & $\begin{array}{c}\text { Abnormal } \\
\text { Pap Smear } \\
\text { N (\%) }\end{array}$ & P-value \\
\hline \multicolumn{5}{|l|}{ Age, years } \\
\hline$\leq 45$ & $90(45.0)$ & $36(36.0)$ & $54(54.0)$ & \multirow[t]{2}{*}{$0.01 *$} \\
\hline$>45$ & $110(55.0)$ & $64(64.0)$ & $46(46.0)$ & \\
\hline \multicolumn{5}{|l|}{ Parity } \\
\hline$\leq 2$ & $182(91.0)$ & $90(90.0)$ & $92(92.0)$ & \multirow[t]{2}{*}{0.62} \\
\hline$>3$ & $18(9.0)$ & $10(10.0)$ & $8(8.0)$ & \\
\hline \multicolumn{5}{|l|}{ Marital status } \\
\hline Non-married & $51(25.5)$ & $24(24.0)$ & $27(27.0)$ & \multirow[t]{2}{*}{0.63} \\
\hline Married & $149(74.5)$ & $76(76.0)$ & $73(73.0)$ & \\
\hline \multicolumn{5}{|l|}{ Smoking } \\
\hline NO & $195(97.5)$ & $99(99.0)$ & $96(96.0)$ & \multirow[t]{2}{*}{0.37} \\
\hline YES & $5(2.5)$ & 1(1.0) & $4(4.0)$ & \\
\hline \multicolumn{5}{|c|}{ Alcohol consumption } \\
\hline NO & $146(73.4)$ & $76(76.8)$ & $70(70.0)$ & \multirow[t]{2}{*}{0.28} \\
\hline YES & $53(26.6)$ & $23(23.2)$ & $30(30.0)$ & \\
\hline \multicolumn{5}{|c|}{ Sex partners, number } \\
\hline 1 & $132(68.0)$ & $73(75.3)$ & $59(60.8)$ & \multirow[t]{2}{*}{$0.03 *$} \\
\hline$>1$ & $62(32.0)$ & $24(24.7)$ & $38(39.2)$ & \\
\hline \multicolumn{5}{|l|}{ Comorbidities } \\
\hline $\mathrm{NO}$ & $111(55.5)$ & $53(53.0)$ & $58(58.0)$ & \multirow[t]{2}{*}{0.48} \\
\hline YES & $89(44.5)$ & $47(47.0)$ & $42(42.0)$ & \\
\hline \multicolumn{5}{|l|}{ History of cancer } \\
\hline $\mathrm{NO}$ & $194(97.0)$ & $96(96.0)$ & $98(98.0)$ & \multirow[t]{2}{*}{0.68} \\
\hline YES & $6(3.0)$ & $4(4.0)$ & $2(2.0)$ & \\
\hline \multicolumn{5}{|c|}{ Family history of cancer } \\
\hline $\mathrm{NO}$ & $129(64.5)$ & $63(63.0)$ & $66(66.0)$ & \multirow[t]{2}{*}{0.66} \\
\hline YES & $71(35.5)$ & $37(37.0)$ & $34(34.0)$ & \\
\hline \multicolumn{5}{|l|}{ Caregivers } \\
\hline $\mathrm{NO}$ & $16(8.0)$ & $11(11.1)$ & $5(5.0)$ & \multirow[t]{2}{*}{0.11} \\
\hline YES & $183(92.0)$ & $88(88.9)$ & $95(95.0)$ & \\
\hline \multicolumn{5}{|l|}{ Religion } \\
\hline Buddhist & $194(97.0)$ & $97(97.0)$ & $97(97.0)$ & \multirow[t]{2}{*}{1} \\
\hline Christian & $6(3.0)$ & $3(3.0)$ & $3(3.0)$ & \\
\hline \multicolumn{5}{|l|}{ Education } \\
\hline None & $6(3.0)$ & $0(0.0)$ & $6(6.0)$ & \multirow[t]{4}{*}{0.07} \\
\hline Primary & $54(27.3)$ & $30(30.6)$ & $24(24.0)$ & \\
\hline Secondary & $59(29.8)$ & $27(27.6)$ & $32(32.0)$ & \\
\hline University & 79 (39.9) & $41(41.8)$ & $38(38.0)$ & \\
\hline Monthly salary, b & & & & \\
\hline$<5,000$ & $59(30.7)$ & $27(28.7)$ & $32(32.7)$ & 0.79 \\
\hline $5,000-9,999$ & $39(20.3)$ & $19(20.2)$ & $20(20.4)$ & \\
\hline $10,000-19,999$ & $39(20.3)$ & $18(19.1)$ & $21(21.4)$ & \\
\hline $20,000-49,999$ & $46(24.0)$ & $24(25.5)$ & $22(22.4)$ & \\
\hline$>50,000$ & 9 (4.7) & $6(6.4)$ & $3(3.1)$ & \\
\hline
\end{tabular}


Table 2. Comparison of HADS-A and HADS-D Scores between Study Groups

\begin{tabular}{lccc}
\hline Outcomes & $\begin{array}{c}\text { Abnormal } \\
\text { Pap Smear }\end{array}$ & $\begin{array}{c}\text { Normal } \\
\text { control }\end{array}$ & p-value \\
\hline HADS-A $\geq 11, \mathrm{~N}(\%)$ & $14(14.0)$ & $3(3.0)$ & $<0.01^{*}$ \\
HADS-D $\geq 11, \mathrm{~N}(\%)$ & $1(1.0)$ & $1(1.0)$ & 1.00 \\
Mean HADS-A \pm SD (min-max) & & \\
$6.6 \pm 3.7(0-20)$ & $4.8 \pm 3.0(0-16)$ & $<0.01^{*}$ \\
Mean HADS-D \pm SD (min-max) & & \\
$3.9 \pm 2.9(0-14)$ & $3.1 \pm 2.6(0-13)$ & 0.05 \\
\hline
\end{tabular}

*SD: Standard deviation; HADS: Hospital Anxiety and Depression Scale; A: Anxiety; D: Depression

group, $14.0 \%$ vs $3.0 \%$. The prevalence of depression was comparable between the groups. The mean HADS-A and HADS-D scores were significantly higher in the abnormal Pap group, 6.6 vs 4.8 and 3.9 vs 3.1, respectively. In multivariable analysis when study group, age, parity, marital status, alcohol consumption, number of sexual partner, co-morbidity, history of cancer, family history of cancer, availability of caregiver, religion, education, salary, route of information regarding the importance of Pap test, frequency of having Pap test, and reason for having the current Pap test were taken into account, only the study group (abnormal Pap $v s$ control) was significantly associated with anxiety $(\mathrm{p}=0.05)$.

Association between demographic/clinical factors and anxiety in participants with abnormal Pap test results was examined. No independent predicting factors for anxiety were identified in this group of patients. There was no significant difference in the mean HADS-A score in participants who had waiting duration from receiving Pap result and colposcopy of less than 1 month $(6.7+3.7)$, 1-2 month $(6.9+3.5)$, and more than 2 months $(5.6+2.0)$ $(\mathrm{p}=0.46)$.

\section{Discussion}

It has been widely reported in the western literature that women with abnormal Pap test undergoing colposcopy had high-level of anxiety (Marteau et al., 1990; Rogstad, 2002; Walsh et al, 2004; Kola and Walsh, 2009). However, information from Asian population, which has significant cultural difference, is lacking. We have demonstrated in this study the significantly higher prevalence of anxiety in women with abnormal Pap test results awaiting colposcopic examination. In addition, we found higher HADS scores for both anxiety and depression subscales among these women. Our finding were consistent with the previous reports with different cultural background. Of note, anxiety can have further adverse effects on psychological conditions during the colposcopic procedure and on health-related quality of life (HRQoL) afterward. It has been shown that colposcopy related pain and discomfort were significantly affected by pre-procedural state anxiety level (Baser et al., 2013). Heinonen et al. has demonstrated that abnormal cervical cytology and colposcopy referral were associated with anxiety and compromised some dimension of HRQoL including sleeping, distress, and sexual activities. However, overall HRQoL was not reduced. It has been stressed that high- level of baseline anxiety were associated with decreased HRQoL (Heinonen et al., 2013).

It is important to note that the screening program per se can have significant adverse psychological impact starting at the time of participation in the program. After screening, level of psychological distress has been shown to increase even if the screening result is negative (Stoate, 1989; Rogstad, 2002). The negative psychological effects including situational anxiety, impaired concentration, tension, and depression, are even more obvious in women with abnormal screening test result necessitating colposcopy, usually together with diagnostic directed cervical biopsy. In this situation, documented causes of anxiety are based on fears of cancer, colposcopy and related procedures, loss of reproductive and sexual function, loss of body image and integrity, loss of dignity, and possibility of transmission of the abnormality to sexual partner. In order to guide development of effective intervention to reduce anxiety and other negative psychological effects among these women, Kola et al. reported that being single, having children, having high trait anxiety level, and anticipating pain and discomfort, were contributing factors for pre-colposcopy psychological distress (Kola and Walsh, 2012). In our study, however, no predicting factor for anxiety could be identified among participants in the abnormal Pap group.

Recently, it was found that patients' problem-focused coping can provide positive physical and spiritual effects on well-being of women with cancer, resulting in moderately high-level of HRQoL, in spite of negative impact of the disease and prolonged treatment. The commonly employed problem-focused coping included acceptance (an active intention to learn to live with disease and adjust to the situation), using emotional support, religion belief, and positive reframing (Tuncay, 2014). The positive psychological impact of feeling at peace and having a sense of meaning in life was also stressed in cancer patients receiving palliative care (Sharifa Ezat et al., 2014). Although these strategies can be effective in coping with chronic deseases like cancer, their use in dealing with anxiety or other negative psychological conditions resulted from abnormal Pap test and related procedures remains questionable given the uncertainty in diagnosis, which, by itself, could provoke anxiety, and also the shorter nature of the situation.

Generally, women that have Pap test done at our institution receive the test results by postal mail in approximately one month. For those who have abnormal result, the need and appointment for colposcopic examination are informed in the letter. There is no physician or health personnel contact before the scheduled colposcopic visit. Therefore, the high prevalence of anxiety in these women was not surprising. Various interventions have been proposed to reduce anxiety in this circumstance. The studied interventions included use of information leaflets, use of information video, counselling session, use of video colposcopy, and listening to music during colposcopy. A recent systematic review has shown that, of those interventions, listening to music during colposcopy significantly reduced anxiety and pain during the procedure. Although, the use of information 
Tanut Jerachotechueantaveechai et al

leaflets did not reduce anxiety, it improved knowledge so could facilitate informed consent process. Also, using information leaflets enhanced quality of life by reducing psychosexual dysfunction (Galaal et al., 2011).

We used the Thai version of HADS as a screening tool for anxiety and depression because of its reliability, validity, simplicity, and good performance in the screening of anxiety and depression in non-psychiatric setting. In general, the cut-off score of $>8$ is accepted as a threshold for both HADS-A and HADS-D as a case finder (Bjelland et al., 2002). However, for the Thai version, the cut-off point of $>11$ used in this study, has been recommended. When tested in 60 in-patients with cancer using the cut-off point of $>11$, the sensitivity and specificity of the anxiety subscale were $100 \%$ and $86 \%$, respectively while the sensitivity and specificity of the depression subscale were $85.7 \%$ and $91.3 \%$, respectively. The internal consistencies appeared good with the Cronbach's alpha coefficient of 0.86 for anxiety subscale and 0.83 for depression subscale (Nilchaikovit et al., 1996). In our study, the Cronbach's alpha coefficient could be considered good $(0.82)$ for the anxiety subscale and satisfactory (0.71) for the depression subscale.

Some study limitations existed. There were baseline imbalances in age and proportion of those having more than one sexual partner between the groups. The multivariable analysis was employed to adjust for these and other possible confounding factors. In addition, the sample size was too small to determine predicting factors for anxiety in women with abnormal Pap test and to examine possible difference in the prevalence of depression between the study groups as suggested by the difference in HADS-D scores.

In conclusion, the prevalence of anxiety in women with abnormal Pap test undergoing colposcopy was significantly higher than that of normal controls. Special attention including thorough counselling, the use of information leaflet, and psychological support should be directed to these women. However, predicting factors for anxiety in this group of women were not discovered.

\section{Acknowledgements}

Faculty of Medicine, Chiang Mai University and the National Research University Project under Thailand's Office of the Higher Education Commission provided funding support for this project.

\section{References}

Baser E, Togrul C, Ozgu E, et al. (2013). Effect of pre-procedural state-trait anxiety on pain perception and discomfort in women undergoing colposcopy for cervical cytological abnormalities. Asian Pac J Cancer Prev, 14, 4053-6.

Bjelland I, Dahl AA, Haug TT, Neckelmann D (2002). The validity of the Hospital Anxiety and Depression Scale. An updated literature review. J Psychosom Res, 52, 69-77.

Galaal K, Bryant A, Deane KH, Al-Khaduri M, Lopes AD (2011). Interventions for reducing anxiety in women undergoing colposcopy. Cochrane Database Syst Rev, 6013.

Heinonen A, Tapper AM, Leminen A, Sintonen H, Roine RP (2013). Health-related quality of life and perception of anxiety in women with abnormal cervical cytology referred for colposcopy: an observational study. Eur J Obstet Gynecol Reprod Biol, 169, 387-91.

Kavanagh AM, Broom DH (1997). Women's understanding of abnormal cervical smear test results: a qualitative interview study. BMJ, 314, 1388-91.

Kola S, Walsh JC (2009). Patients' psychological reactions to colposcopy and LLETZ treatment for cervical intraepithelial neoplasia. Eur J Obstet Gynecol Reprod Biol, 146, 96-9.

Kola S, Walsh JC (2012). Determinants of pre-procedural state anxiety and negative affect in first-time colposcopy patients: implications for intervention. Eur J Cancer Care, 21, 469-76.

Marteau TM, Walker P, Giles J, Smail M (1990). Anxieties in women undergoing colposcopy. BJOG, 97, 859-61.

Nilchaikovit T, Lotrakul M, Phisansuthideth U (1996). Development of Thai version of Hospital Anxiety and Depression Scale in cancer patients. J Psychiatr Assoc Thai, 41, 18-30.

Quinn M, Babb P, Jones J, Allen E (1999). Effect of screening on incidence of and mortality from cancer of cervix in England: evaluation based on routinely collected statistics. $B M J, 318,904-8$.

Rogstad KE (2002). The psychological impact of abnormal cytology and colposcopy. BJOG, 109, 364-8.

Sharifa Ezat WP, Fuad I, Hayati Y, Zafar A, Wanda Kiyah GA (2014). Observational study on patient's satisfactions and quality of life (QoL) among cancer patients receiving treatment with palliative care intent in a tertiary hospital in Malaysia. Asian Pac J Cancer Prev, 15, 695-701.

Stoate HG (1989). Can health screening damage your health? J $R$ Coll Gen Pract, 39, 193-5.

Tuncay T (2014). Coping and quality of life in Turkish women living with ovarian cancer. Asian Pac J Cancer Prev, 15, 4005-12.

Walsh JC, Curtis R, Mylotte M (2004). Anxiety levels in women attending a colposcopy clinic: a randomised trial of an educational intervention using video colposcopy. Patient Educ Couns, 55, 247-51.

Willoughby BJ, Faulkner K, Stamp EC, Whitaker CJ (2006). A descriptive study of the decline in cervical screening coverage rates in the North East and Yorkshire and the Humber regions of the UK from 1995 to 2005. J Public Health, 28, 355-60.

Zigmond AS, Snaith RP (1983). The hospital anxiety and depression scale. Acta Psychiatr Scand, 67, 361-70. 